\title{
Renaming schizophrenia to reduce stigma: comparison with the case of bipolar disorder
}

Nell Ellison, Oliver Mason and Katrina Scior

\begin{abstract}
Summary
Renaming disorders to change public beliefs and attitudes remains controversial. This study compared the potentially destigmatising effects of renaming schizophrenia with the effects of renaming bipolar disorder by comparing the labe 'schizophrenia' to 'integration disorder', and 'bipolar disorder' to 'manic depression', in 1621 lay participants. 'Bipolar disorder' was associated with less fear and social distance than 'manic
\end{abstract}

depression'. 'Integration disorder' was associated with increased endorsement of a biopsychosocial cause and reduced attributions of dangerousness but also increased social distance, highlighting the complex effects renaming has on stigma.

\section{Declaration of interest}

None.
Whether schizophrenia should be renamed to help reduce stigma has sparked debate among the scientific community. ${ }^{1-3}$ Some argue that changing the name would help reduce iatrogenic stigma, as schizophrenia has connotations of disease and 'split-mind. ${ }^{3}$ Others maintain that stigma is due to the public's ignorance and fear of people with mental illness ${ }^{2}$ and better education, not a name change, is what is necessary. In 2002, Japan changed their term for schizophrenia from 'split-mind-disease' to 'integration disorder' in an effort to change public attitudes. ${ }^{4,5}$ Similarly, although not directly aimed at changing attitudes, 'manic depression' was officially changed to 'bipolar disorder' in 1980. This change has been cited as an example of where changing terminology has helped to reduce stigma. ${ }^{6}$ However, there is little empirical evidence regarding renaming; whereas one study exploring the effect of the Japanese name change reported a decrease in endorsement of the stereotype 'criminal' among college students, ${ }^{5}$ two studies using small college student samples, in China and Canada, found no difference between different terms presented for schizophrenia. ${ }^{7,8}$ There have been no general population studies exploring the effect of different labels for schizophrenia on public beliefs and attitudes, and no studies exploring the effect of renaming on all components of public stigma (i.e. cognitive, emotional and behavioural reactions). No studies have explored the effect of the name change in bipolar disorder on public beliefs and attitudes. This study aimed to address these gaps in the literature for both disorders.

\section{Method}

We recruited 1621 UK residents using email, flyers and social networking sites using an incentivised form of snowballing ${ }^{9}$ (see online Table DS1 for sociodemographic characteristics). Of those who accessed the site, $79.7 \%$ completed the online survey. We used an experimental randomised cross-sectional design. Each participant received two vignettes: one met DSM-IV ${ }^{10}$ and ICD- $10^{11}$ criteria for schizophrenia and was labelled either schizophrenia or integration disorder, and the other met DSM-IV and ICD-10 criteria for bipolar disorder and was labelled either bipolar disorder or manic depression. Label pairing and presentation order were counterbalanced and participants randomly assigned. Vignettes were reviewed by five experts for the purpose of masked diagnostic allocation and to ensure they were deemed representative of the target disorder and of equal severity.

After being presented with each vignette, respondents completed questionnaires covering five aspects of public stigma: causal beliefs, prognosis, emotional reactions, stereotypes and behaviour (social distance). Derivations of the vignettes and the public stigma questionnaire scales are given in the online supplement. In the present sample, alpha coefficients were good to excellent for all scales. To assess the influence of different diagnostic labels on beliefs and attitudes, $2 \times 2 \times 2$ between-subjects ANOVAs were conducted for each vignette separately. The inclusion of presentation order and pairing as fixed factors allowed for the assessment of any interaction effect with the effect of label. There was no interaction, indicating that the effect of label did not vary depending on which label was presented first or which label it was paired with. All $P$-values were Bonferroni corrected for tests carried out within each questionnaire.

\section{Results}

\section{'Bipolar disorder' v. 'manic depression'}

There was a significant difference between the two labels on all causal beliefs, with psychosocial causes being ascribed more to the label manic depression than to bipolar disorder (Table 1 and online Table DS2 for means and standard deviations). Biomedical causes were ascribed more to the label bipolar disorder than to manic depression, and fate causes were ascribed more to the label manic depression than to bipolar disorder. The label manic depression elicited more fear and a greater desire for social distance than bipolar disorder. There was no difference between the two labels on prognosis without treatment or under optimal treatment; compassion or anger; or on the stereotypes of dangerousness, dependency, or intelligence and creativity.

\section{Schizophrenia' $v$. 'integration disorder'}

Psychosocial causes were ascribed more to the label integration disorder than schizophrenia (Table 1 and online Table DS2). The stereotype of dangerousness was ascribed more to the label schizophrenia than to the label integration disorder. Conversely, the label integration disorder elicited a greater desire for social distance than schizophrenia. There was no difference between the two labels on biomedical or fate causal beliefs; prognosis without treatment or under optimal treatment; fear, compassion, or anger; or on the stereotypes of dependency, or intelligence and creativity.

\section{Discussion}

The influence of different diagnostic labels on stigma appears complex. 'Bipolar disorder' was associated with less stigma on some domains, with it reducing fear and desire for social distance. 'Integration disorder' had mixed effects, with it increasing endorsement of psychosocial causes and reducing attributions 
Table 1 Effect of different diagnostic labels for bipolar disorder and schizophrenia ${ }^{a}$

\begin{tabular}{|c|c|c|c|c|}
\hline & \multicolumn{2}{|c|}{ Bipolar disorder } & \multicolumn{2}{|c|}{ Schizophrenia } \\
\hline & $f$ & $d$ & $f$ & $d$ \\
\hline \multicolumn{5}{|l|}{ Causal beliefs } \\
\hline Psychosocial & $10.19^{* *}$ & 0.16 & $8.68^{* *}$ & 0.14 \\
\hline Biomedical & $12.85^{\star * *}$ & 0.20 & 5.48 & - \\
\hline Fate & $6.73^{*}$ & 0.14 & 3.03 & - \\
\hline \multicolumn{5}{|l|}{ Prognosis } \\
\hline Without treatment & 0.56 & - & 0.99 & - \\
\hline Under optimal treatment & 0.38 & - & 0.02 & - \\
\hline \multicolumn{5}{|l|}{ Emotional reactions } \\
\hline Fear & $6.69 *$ & 0.14 & 2.19 & - \\
\hline Compassion & 0.17 & - & 1.07 & - \\
\hline Anger & 0.04 & - & 0.44 & - \\
\hline \multicolumn{5}{|l|}{ Stereotypes } \\
\hline Dangerousness & 0.78 & - & $9.28 * *$ & 0.15 \\
\hline Dependency & 4.87 & - & 1.21 & - \\
\hline Intelligence/creativity & 0.06 & - & 3.96 & - \\
\hline Social distance & $9.36 * * *$ & 0.16 & $5.43^{\star}$ & 0.13 \\
\hline \multicolumn{5}{|c|}{$\begin{array}{l}\text { a. Bipolar disorder: } n=1569-1579 \text {; schizophrenia: } n=1566-1578 ; \text { d.f. }=1 \text {, error } \\
\text { d.f. }=1558-1572 \text {. } \\
{ }^{*} P<0.05 \text {, }{ }^{* *} P<0.01,{ }^{* *} P<0.001 \text {. }\end{array}$} \\
\hline
\end{tabular}

dangerousness, while simultaneously increasing desire for social distance. These significant findings all showed a small effect size at best, yet the $1.6-3.4 \%$ change observed on these measures compares favourably with Time to Change, the UK's largest anti-stigma campaign, which demonstrated a $1.4 \%$ improvement on measures of attitudes and social distance since $2008 .{ }^{12}$ One aim in the Japanese renaming of schizophrenia was to promote a biopsychosocial model of causality. ${ }^{4}$ In our study, integration disorder was ascribed more to psychosocial and less to biomedical causes, suggesting this objective may be achieved if it were to be renamed. The belief that people with schizophrenia are dangerous is consistently cited as detrimental to their inclusion in society. Findings suggest that, independent of behaviour, the term schizophrenia may have a role to play in perpetuating this stereotype. The label integration disorder had a negative effect on social distance. Van $\mathrm{Os}^{3}$ proposed that the term integration disorder may paradoxically induce stigma because the public cannot relate to a universal psychological function of 'integration'. Research is therefore needed to explore alternative labels for schizophrenia, such as 'salience syndrome.' Integration disorder was also the less familiar diagnosis, and familiarity is known to reduce social distance. ${ }^{13}$

The more positive attitudes associated with 'bipolar disorder' may be due to the impact it had on causal beliefs. That is, endorsement of biomedical beliefs have been found to have a positive effect on stigma, ${ }^{14}$ whereas endorsement of fate causal beliefs have a negative effect. ${ }^{15}$ It may also simply represent an effect of iatrogenic stigma, with manic depression evoking descriptions of people as 'maniacs'. Renaming may also have indirect effects on public stigma through the reduction of internalised stigma. Specifically, it may have allowed people to reject the negative stereotypes attached to manic depression, subsequently promoting disclosure and therefore increasing contact between people with bipolar disorder and the general population. It may also have made those being disclosed to more receptive to education by reducing initial assumptions. This study does not support the assertion that any benefits of renaming are short-lived as 'bipolar disorder' continues to be less stigmatised. Indeed, any positive effects of renaming through changes in internalised stigma, or through its interaction with education, are likely to take time to become apparent.

This is the largest study to explore renaming and stigma, and although the ethnicity of the sample was broadly representative of the UK population, the sample was predominantly female and educated to degree level, which may limit generalisability. Importantly, though, neither gender nor educational attainment predicted scores on social distance in the present sample.

The negative effect the term integration disorder had on social distance suggests any decisions to rename should be made with caution. However, a decision not to rename may overlook an important opportunity to tackle damaging stereotypes and promote a biopsychosocial model of causality. Finally, service users and families have been campaigning for over 30 years to have the term for schizophrenia changed. ${ }^{4}$ It is of paramount importance that this is not ignored in any decision regarding renaming.

Nell Ellison, MA(Hons), DclinPsy, Oliver Mason, BSc, DClinPsy, PhD, Katrina Scior, BSC, DClinPsych, PhD, Research Department of Clinical, Educational and Health Psychology, University College London, London, UK

Correspondence: Nell Ellison, University College London, 66C Whitehall Park, London N19 3TN, UK. Email: nell.ellison.10@ucl.ac.uk

First received 9 Feb 2014, final revision 21 Sep 2014, accepted 29 Sep 2014

\section{References}

1 Levin T. Schizophrenia should be renamed to help educate patients and the public. Int J Soc Psychiatry 2006; 52: 324-31.

2 Lieberman JA, First MB. Renaming schizophrenia. BMJ 2007; 334: 108

3 van Os J. 'Salience syndrome' replaces 'schizophrenia' in DSM-V and ICD-11: psychiatry's evidence-based entry into the 21st century? Acta Psychiatr Scand 2009; 120: 363-72.

4 Sato M. Renaming schizophrenia: Japanese perspective. World Psychiatry 2006; 5: 53-5.

5 Takahashi $\mathrm{H}$, Ideno $\mathrm{T}$, Okubo $\mathrm{S}$, Matsui $\mathrm{H}$, Takemura $\mathrm{K}$, Matsuura $\mathrm{M}$, et al Impact of changing the Japanese term for "schizophrenia" for reasons of stereotypical beliefs of schizophrenia in Japanese youth. Schizophr Res 2009; 112: $149-52$

6 Kingdon D, Hansen L, Kinoshita Y, Naeem F, Rathod S, Swelam M, et al. Schizophrenia can and should be renamed. Rapid response to Leiberman \& First 'Renaming Schizophrenia'. BMJ 2007; 334: 108 (http://www.bmj.com/ content/334/7585/108?tab = response-form).

7 Chung KF, Chan JH. Can a less pejorative Chinese translation for schizophrenia reduce stigma? A study of adolescents' attitudes toward people with schizophrenia. Psychiatry Clin Neurosci 2004; 58: 507-15.

8 Tranulis C, Lecomte T, El-Khoury B, Lavarenne A, Brodeur-Côté D. Changing the name of schizophrenia: patient perspectives and implications for DSM-V. PLOS One 2013; 8: 2.

9 Gardner B. Incentivised snowballing: a method for recruiting to internetbased research studies. Psychologist 2009; 22: 768-9.

10 American Psychiatric Association. Diagnostic and Statistical Manual of Mental Disorder (4th edn) (DSM-IV). APA, 1994

11 World Health Organization. The ICD-10 Classification of Mental and Behavioural Disorders: Clinical Descriptions and Diagnostic Guidelines. WHO, 1992.

12 Evans-Lacko S, Henderson C, Thornicroft G. Public knowledge, attitudes and behaviour regarding people with mental illness in England 2009-2012. Br J Psychiatry 2013; 202 (s55): s51-7.

13 Angermeyer MC, Matschinger $\mathrm{H}$, Corrigan PW. Familiarity with mental illness and social distance from people with schizophrenia and major depression: testing a model using data from a representative population survey. Schizophr Res 2004; 69: 175-82.

14 Corrigan PW, River LP, Lundin RK, Wasowski KU, Campion J, Mathisen J, et al. Stigmatizing attributions about mental illness. J Community Psychol 2000; 28: $91-102$.

15 Mulatu MS. Perceptions of mental and physical illnesses in north-western Ethiopia: causes, treatments, and attitudes. J Health Psychol 1999; 4: 531-49. 